\title{
Quarkonium production measured by the STAR experiment
}

\section{Leszek Kosarzewski*t}

Faculty of Nuclear Sciences and Physical Engineering, Czech Technical University in Prague

E-mail: kosarlesefjei.cvut.cz

Properties of the Quark Gluon Plasma, created in heavy ion collisions, can be studied by measuring production of various quarkonium states. Each state has a different binding energy and is expected to dissociate at a different temperature in the plasma. By comparing such a sequential suppression pattern to the model calculations, thermodynamical properties of the medium can be extracted. Cold nuclear matter effects however also play a role and can be studied in $\mathrm{p}+\mathrm{A}$ or $\mathrm{d}+\mathrm{A}$ collisions. Finally, quarkonium production in $\mathrm{p}+\mathrm{p}$ collisions has to be measured in order to understand the quarkonium production mechanism.

In these proceedings, we present an overview of $J / \psi$ and $\Upsilon$ production measured with the STAR experiment. Results from different colliding systems $(p+p, p+A u, A u+A u$ and $U+U)$ are discussed.

European Physical Society Conference on High Energy Physics - EPS-HEP2019 -

10-17 July, 2019

Ghent, Belgium

\footnotetext{
* Speaker.

${ }^{\dagger}$ For the STAR Collaboration
} 


\section{Introduction}

In relativistic heavy ion collisions a state of matter containing deconfined quarks and gluons is created. It's properties can be studied using quarkonium states, which dissociate when a high enough temperature is reached as a result of Debye-like screening of color charges [1]. This happens if the Debye screening length $r_{\text {Debye }}$ is smaller than the radius of a particular quarkonium state. Increasing the temperature of the plasma causes the Debye screening length to decrease as $r_{\text {Debye }} \propto T^{-1}$. Since each quarkonium state has a different binding energy and radius, it should dissociate at a different temperature leading to a sequential suppression pattern for different quarkonium states [2]. Such a suppression is measured with respect to the yield in $p+p$ collisions, by calculating the nuclear modification factor:

$$
R_{A A}=\frac{1}{\left\langle N_{\text {coll }}\right\rangle} \frac{\mathrm{d}^{2} N_{A+A} / \mathrm{d} p_{T} \mathrm{~d} y}{\mathrm{~d}^{2} N_{p p} / \mathrm{d} p_{T} \mathrm{~d} y}
$$

which is a ratio of quarkonium production yield in $\mathrm{A}+\mathrm{A}$ collisions to the yield in $\mathrm{p}+\mathrm{p}$ collisions scaled by the mean number of binary collisions in $\mathrm{A}+\mathrm{A}$. A suppression of $J / \psi$ production has been observed at SPS [3], RHIC [4], and LHC [5] as well as for $\Upsilon$ at RHIC [6] and the sequential suppression of $\Upsilon$ states at LHC [7].

In addition to the suppression discussed above, there are other effects, which complicate the interpretation. There might be a feed-down contribution from the radiative or hadronic decays of excited quarkonium states to the measured yield. Furthermore, Cold nuclear matter (CNM) effects also play a role. These include modifications (shadowing, anti-shadowing) to the parton distributions (nPDF) in a nucleus compared to a free nucleon. Further CNM effects can come from to interactions with comoving hadrons or nuclear absorption. Such effects can be studied in $\mathrm{p}+\mathrm{A}$ or $\mathrm{d}+\mathrm{A}$ collisions and estimated with the help of theoretical calculations.

Finally, quarkonium production needs to be measured in $\mathrm{p}+\mathrm{p}$ collisions, which serves as a reference for all the above mentioned studies. This also helps to understand the quarkonium production mechanism, which is not yet fully understood.

\section{Quarkonium measurement with the STAR experiment}

The STAR experiment is well suited to detecting charged particles in a wide solid angle $|\eta|<1$ and $0<\phi<2 \pi$. It consists of a number of detector subsystems. The Time Projection Chamber (TPC) is used for particle identification based on ionization energy loss $\frac{d E}{d x}$ and tracking. The Barrel Electromagnetic Calorimeter (BEMC) is used for electron identification and serves to trigger on high- $p_{T}$ electrons. The Time of Flight Detector (TOF) is also used for particle identification. The TPC, BEMC and TOF are enclosed in a solenoid magnet, producing a $B=0.5 \mathrm{~T}$ field. The Muon Telescope Detector (MTD) is located just outside the magnet, which acts here as an absorber for hadrons, and provides muon identification and triggering in $|\eta|<0.5$ with $45 \%$ azimuthal coverage.

Thanks to the STAR trigger system, studies of rare processes like production of $J / \psi$ and $\Upsilon$ are possible with high precision. A BEMC-based trigger is fired when a high- $p_{T}$ electron hits one of the BEMC towers (cells). On the other hand the dimuon trigger requires a coincidence of 2 hits in the MTD to happen within a short time window after bunch crossing. 
At STAR the quarkonium candidates in the dielectron channel are reconstructed by finding $e^{+} e^{-}$pair candidates and projecting their tracks to hits in BEMC. At least one is required to match the hit, which fired the trigger. In the dimuon channel, both of the candidate tracks are required to match MTD hits which fired the dimuon trigger.

\section{Quarkonium production in $\mathbf{p}+\mathbf{p}$ collisions at $\sqrt{s}=200,500$ and $510 \mathrm{GeV}$}

The inclusive $J / \psi$ cross section has been measured by the STAR experiment in p+p collisions at $\sqrt{s}=200,500$ and $510 \mathrm{GeV}[8,9]$. The measurements at $\sqrt{s}=500$ and $510 \mathrm{GeV}$ for inclusive $J / \psi$ are shown in Fig. 1 using both the dielectron and diumon decay channels. Figure 2 shows the same results divided by the result of a Levy fit to the data. These are compared to CGC+NRQCD [10], NLO NRQCD [11] and Improved Color Evaporation Model (ICEM) [12] calculations after adding the $B \rightarrow J / \psi$ feed-down contribution from a FONLL calculation $[13,14]$.

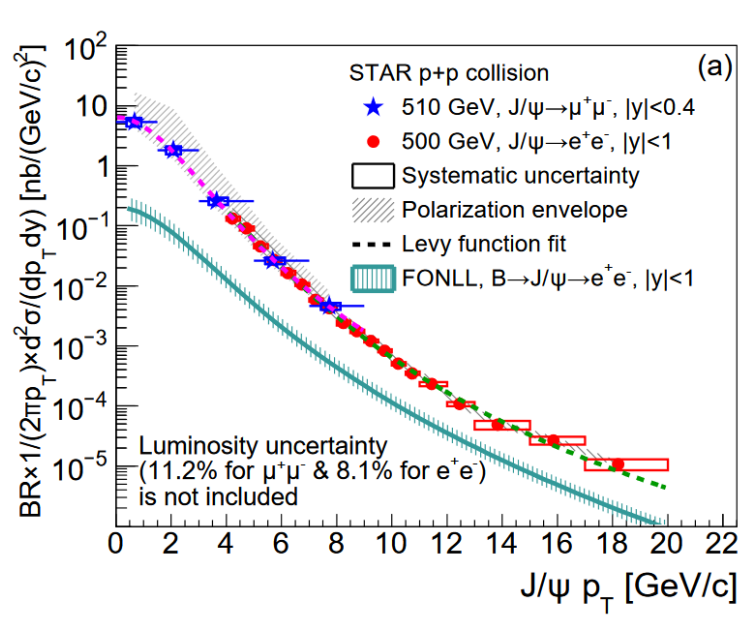

Figure 1: Cross section for inclusive $J / \psi$ production vs. $p_{T}$ measured in $\mathrm{p}+\mathrm{p}$ collisions at $\sqrt{s}=500 \mathrm{GeV}$ and $\sqrt{s}=510 \mathrm{GeV}$ [9]. FONLL calculation of $B \rightarrow$ $J / \psi$ feed-down contribution is also shown (teal curve). The data are fitted with a Levy function (dashed line).

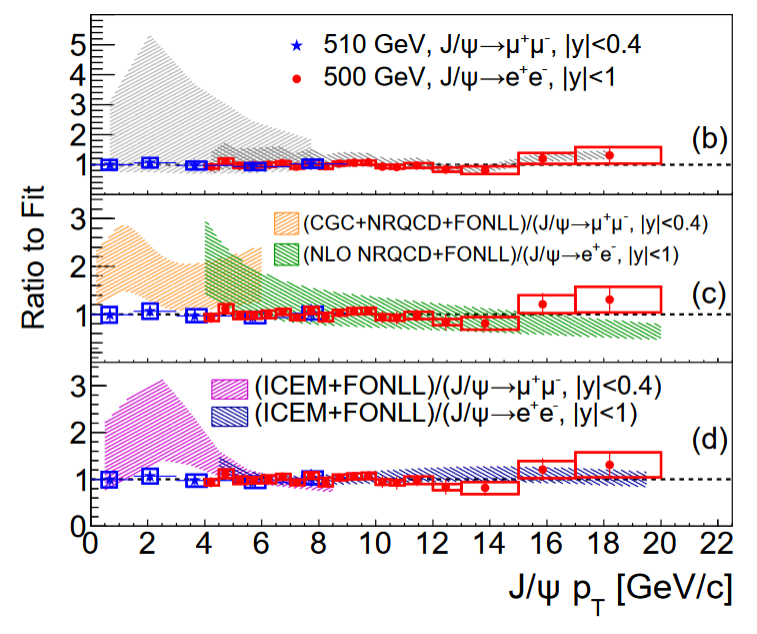

Figure 2: Ratio of the $J / \psi$ cross section from Fig. 1 [9] to the Levy fit. The data are compared to CGC+NRQCD [10] and NLO NRQCD [11] model calculations and ICEM model [12] calculations. The $B \rightarrow J / \psi$ feed-down contribution is included in all model calculations.

The $\Upsilon(1 S), \Upsilon(2 S+3 S)$ and combined $\Upsilon(1 S+2 S+3 S)$ cross sections have been measured in $\mathrm{p}+\mathrm{p}$ collisions at $\sqrt{s}=500 \mathrm{GeV}$ through the dielectron decay channel. They are presented in Fig. 3 . These results are compared to CGC+NRQCD [15, 10] and Color Evaporation Model [16] (CEM) calculations.

Overall, the CEM [17] or ICEM [12] reasonably well describe both the $J / \Psi$ and $\Upsilon$ production in data. However, the ICEM model calculation for $J / p s i$ is on the edge of the data uncertainty band for $p_{T}<4 \mathrm{GeV} / \mathrm{c}$. The $J / \psi$ measurement for $p_{T}>4 \mathrm{GeV} / \mathrm{c}$ is also well described by the NLO NRQCD [11] calculation. On the other hand, the CGC+NRQCD [10] calculation is on the edge of the data uncertainty band for $J / \psi$ and significantly above the data in the low- $p_{T}$ region for $\Upsilon(1 S)$. 


\section{Quarkonium production in $\mathbf{p}+\mathbf{A}$ collisions at $\sqrt{s_{N N}}=200 \mathrm{GeV}$}

Both the $J / \psi$ and $\Upsilon R_{p A u}$ have been measured at $\sqrt{s_{N N}}=200 \mathrm{GeV}$ through the dimuon and dielectron channels, respectively. Figure 4 shows preliminary results on $\Upsilon R_{p A}$, which are compared to previous results from $\mathrm{d}+\mathrm{Au}$ collisions at $\sqrt{s_{N N}}=200 \mathrm{GeV}$ [6] and model calculations incorporating energy loss or nPDF [18] or both [19] effects. These new results have an improved precision over the previous ones and suggest $\Upsilon$ suppression in $\mathrm{p}+\mathrm{Au}$ collisions. The new STAR data are systematically overestimated by the models, but are on the edge of the model uncertainty band.

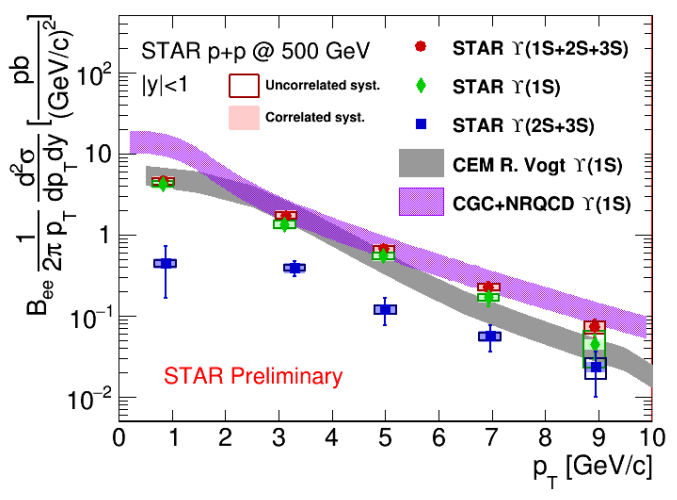

Figure 3: $\quad$ Inclusive $\Upsilon(1 S+2 S+3 S), \Upsilon(1 S)$ and $\Upsilon(2 S+3 S)$ cross section vs. $p_{T}$ compared to CGC+NRQCD $[15,10]$ and CEM model [16] calculations.

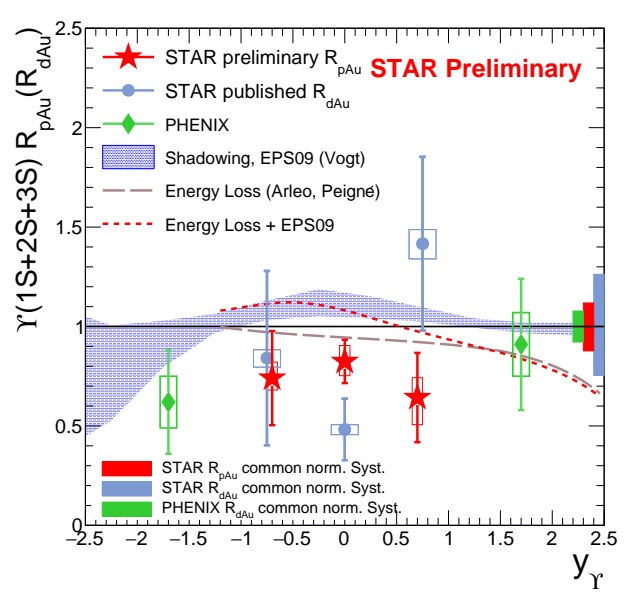

Figure 4: Nuclear modification factor $R_{p A u}\left(R_{d A u}\right)$ vs. rapidity $y$. STAR $\Upsilon$ data in $\mathrm{p}+\mathrm{Au}$ and $\mathrm{d}+\mathrm{Au}$ collisions [6] are compared to PHENIX data [20] and model predictions including nPDF [18] and energy loss with nPDF [19] effects.

\section{Quarkonium production in $\mathbf{A}+\mathbf{A}$ collisions at $\sqrt{s_{N N}}=200 \mathrm{GeV}$}

The $J / \psi$ nuclear modification factor $R_{A A}$ has been measured in both the dielectron and dimuon channels in $\mathrm{Au}+\mathrm{Au}$ collisions at $\sqrt{s_{N N}}=200 \mathrm{GeV}$. The $R_{A A}$ has also been measured in the dielectron channel in $\mathrm{U}+\mathrm{U}$ collisions at $\sqrt{s_{N N}}=193 \mathrm{GeV}$. The new measurement in the dielectron channel are presented in Fig. 5 [21] for different collision centralities. A strong increase has been observed at very low $p_{T}<0.1 \mathrm{GeV} / \mathrm{c}$, which is likely caused by coherent photon-nucleus interactions. On the other hand at $p_{T} \sim 1 \mathrm{GeV} / \mathrm{c}$ a suppression, due to dissociation in QGP and possible CNM effects, is observed.

Figure 6 [22] shows $J / \psi R_{A A}$ in $\mathrm{Au}+\mathrm{Au}$ collisions vs. number of participant nucleons $N_{\text {part }}$ measured in the dimuon channel along with ALICE measurements. The data are compared to Rapp's [23] (TAMU) and Tsinghua group calculations [24, 25] which take into account both dissociation and regeneration effects, and also to the Statistical Hadronization Model (SHM) [26]. Rapp's model includes CNM effects and uses a T-matrix based approach to describe the in-medium dynamics of the $c \bar{c}$ pair. The Tsinghua model does not include the CNM effects. It describes the 
$J / \psi$ behavior in the hot medium using transport equations. Finally, the SHM model assumes thermal production of $J / \psi$ at the time of hadronization including the regeneration effect. All models agree with the data well, with the exception of the SHM model, which gives slightly worse description.

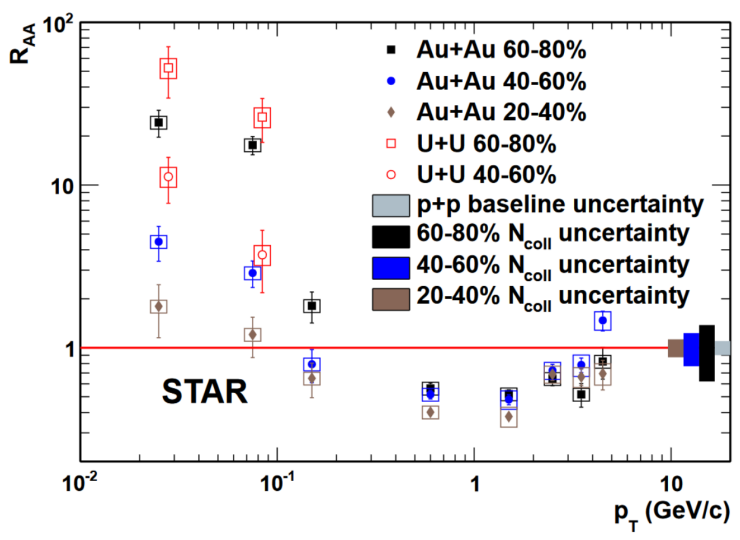

Figure 5: Nuclear modification factor $R_{A A}$ for $J / \psi$ at very low $p_{T}<0.2 \mathrm{GeV} / \mathrm{c}$ [21]. The data are shown for different systems $\mathrm{Au}+\mathrm{Au}$ and $\mathrm{U}+\mathrm{U}$ as well as different collision centralities.

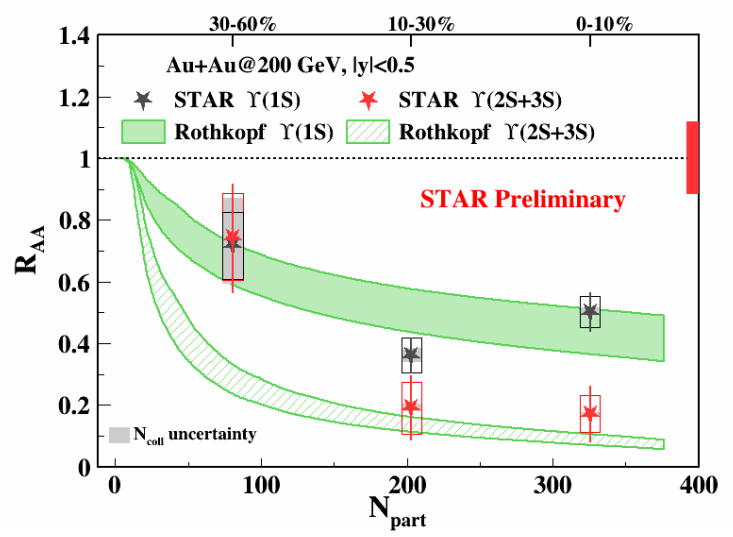

Figure 7: Nuclear modification factor $R_{A A}$ vs. $N_{\text {part }}$ for $\Upsilon(1 S)$ and $\Upsilon(2 S+3 S)$ compared to Rothkopf's model calculation [27].

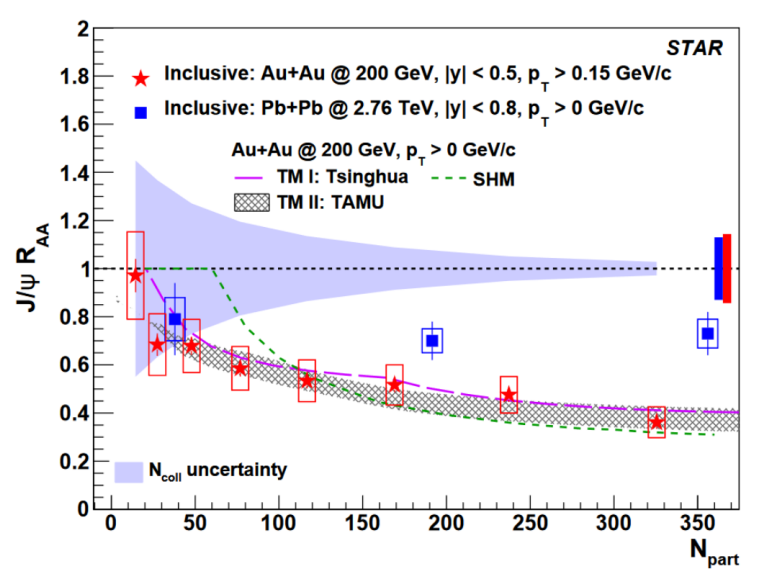

Figure 6: Nuclear modification factor $R_{A A}$ vs. $N_{\text {part }}$ for $J / \psi$ measured by STAR [22] compared to ALICE results and Rapp's model calculation (TAMU) [23]. Statistical Hadronization Model (SHM) [26] and Tsinghua Model [24, 25] are also shown.

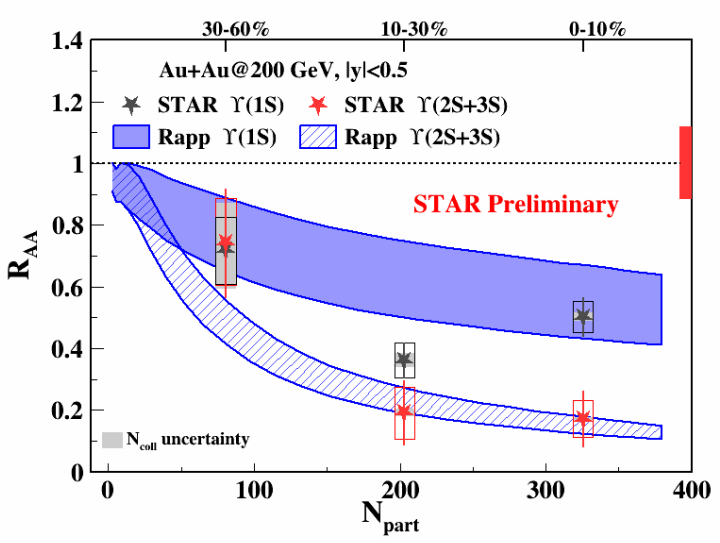

Figure 8: Nuclear modification factor $R_{A A}$ vs. $N_{\text {part }}$ as in Fig. 7 compared to Rapp's model [28].

Figure 7 and Fig. 8 show the $\Upsilon(1 S)$ and $\Upsilon(2 S+3 S)$ nuclear modification factor $R_{A A}$ vs. $N_{\text {part }}$ resulting from combined measurements in the dielectron and dimuon channels. The STAR data are shown along with the model calculations by Rothkopf [27] and Rapp [28]. In Rothkopf's model the $Y$ behavior in the QGP medium is described using potentials from lattice QCD calculations. The effects of CNM or regeneration are not included in this model. The data are well described by both models, except for Rothkopf's model for $\Upsilon(2 S+3 S)$ in the $30-60 \%$ centrality interval. 


\section{Summary}

STAR has performed a comprehensive set of measurements of $J / \psi$ and $\Upsilon$ production in different collision systems: $p+p, p+A u, A u+A u, U+U$. All the data have been compared to relevant model calculations. The quarkonium production models: CEM, ICEM and NLO NRQCD describe the $\mathrm{p}+\mathrm{p}$ data reasonably well. CGC+NRQCD calculations are above the STAR results at low $p_{T}$. In $\mathrm{p}+\mathrm{Au}$ collisions the data indicate a suppression of $\Upsilon$ and the models, while on the edge of data uncertainty band, tend to overestimate the data. The $J / \psi$ and $\Upsilon R_{A A}$ vs. $N_{\text {part }}$ measured by STAR are well described by the models compared: Rapp's and Tsinghua models for $J / \psi$ and Rapp's for $\Upsilon$. However, SHM for $J / \psi$ at low $N_{\text {part }}$ and Rothkopf's model for $\Upsilon(2 S+3 S)$ in $30-60 \%$ centrality interval show deviations from the data. A strong enhancement of $J / \psi$ production is observed at low $p_{T}<0.1 \mathrm{GeV} / \mathrm{c}$, which can be explained by coherent photonuclear production in non-central $\mathrm{Au}+\mathrm{Au}$ and $\mathrm{U}+\mathrm{U}$ collisions.

\section{Acknowledgements}

Supported by the project LTT18002 of the Ministry of Education, Youth and Sport of the Czech Republic.

\section{References}

[1] T. Matsui and H. Satz, J/ $\psi$ suppression by quark-gluon plasma formation, Physics Letters B 178 (1986) 416 .

[2] S. Digal, P. Petreczky and H. Satz, Quarkonium feed-down and sequential suppression, Physical Review D 64 (2001) 094015 [hep-ph / 0106017 ].

[3] Alessandro, B. and others, A New measurement of J/ $\psi$ suppression in Pb-Pb collisions at 158-GeV per nucleon, The European Physical Journal C C39 (2005) 335.

[4] Adamczyk, L. et. al, $J / \psi$ production at low $p_{T}$ in $A u+A u$ and $C u+C u$ collisions at $\sqrt{s_{N N}}=200 \mathrm{GeV}$ at STAR, Physical Review C 90 (2014) 024906 [1310 . 3563].

[5] Adam, J. et al, Inclusive, prompt and non-prompt $\mathrm{J} / \psi$ production at mid-rapidity in $\mathrm{Pb}$ - $\mathrm{Pb}$ collisions at $\sqrt{s_{N N}}=2.76 \mathrm{TeV}$, Journal of High Energy Physics 2015 (2015) 51 [1504.07151].

[6] L. Adamczyk et. al, Suppression of $\Upsilon$ production in $d+A u$ and $A u+A u$ collisions at $\sqrt{s_{N N}}=200 \mathrm{GeV}$, Physics Letters B 735 (2014) 127 [1312 .3675].

[7] Chatrchyan, S. et al, Observation of sequential Upsilon suppression in PbPb collisions, Physical Review Letters 109 (2012) 222301 [1208 . 2826].

[8] J. Adam et. al, $J / \psi$ production cross section and its dependence on charged-particle multiplicity in $p+p$ collisions at $\sqrt{s}=200 \mathrm{GeV}$, Physics Letters B 786 (2018) 87 [1805.03745].

[9] J. Adam et al., Measurements of the transverse-momentum-dependent cross sections of $J / \psi$ production at mid-rapidity in proton+proton collisions at $\sqrt{s}=510$ and $500 \mathrm{GeV}$ with the STAR detector, Physical Review D 100 (2019) 052009 [1905.06075].

[10] Y.-Q. Ma and R. Venugopalan, Comprehensive Description of J/ $\psi$ Production in Proton-Proton Collisions at Collider Energies, Physical Review Letters 113 (2014) 192301 [1408 . 4075]. 
[11] Y.-Q. Ma, K. Wang and K.-T. Chao, $J / \psi\left(\psi^{\prime}\right)$ production at the Tevatron and LHC at $O\left(\alpha_{s}^{4} v^{4}\right)$ in nonrelativistic QCD, Physical Review Letters 106 (2011) 042002 [1 009.3655 ].

[12] Y.-Q. Ma and R. Vogt, Quarkonium Production in an Improved Color Evaporation Model, Physical Review D 94 (2016) 114029 [1609.06042].

[13] M. Cacciari, S. Frixione, N. Houdeau, M. L. Mangano, P. Nason and G. Ridolfi, Theoretical predictions for charm and bottom production at the LHC, Journal of High Energy Physics 2012 (2012) 137 [1205.6344].

[14] M. Cacciari, M. L. Mangano and P. Nason, Gluon PDF constraints from the ratio of forward heavy-quark production at the LHC at $\sqrt{s}=7$ and $13 \mathrm{TeV}$, The European Physical Journal $\mathrm{C} 75$ (2015) 610 [1507.06197].

[15] H. Han, Y.-Q. Ma, C. Meng, H.-S. Shao, Y.-J. Zhang and K.-T. Chao, $\Upsilon(n S)$ and $\chi_{b}(n P)$ production at hadron colliders in nonrelativistic QCD, Physical Review D 94 (2016) 014028 [1410.8537].

[16] R. Vogt, Shadowing effects on J/psi and Upsilon production at energies available at the CERN Large Hadron Collider, Physical Review C 92 (2015) 034909 [1507. 04418 ].

[17] A. D. Frawley, T. Ullrich and R. Vogt, Heavy flavor in heavy-ion collisions at RHIC and RHIC II, Physics Reports 462 (2008) 125 [0806.1013].

[18] R. Vogt, private communication, 2014.

[19] F. Arleo and S. Peigné, Heavy-quarkonium suppression in p-A collisions from parton energy loss in cold QCD matter, Journal of High Energy Physics 2013 (2013) 122 [1212 . 0434 ].

[20] A. Adare et al., $\Upsilon(1 S+2 S+3 S)$ production in $d+A u$ and $p+p$ collisions at $\sqrt{s_{N N}}=200 \mathrm{GeV}$ and cold-nuclear-matter effects, Physical Review C 87 (2013) 044909 [1211. 4017 ].

[21] J. Adam et al., Observation of excess $J / \psi$ yield at very low transverse momenta in Au+Au collisions at $\sqrt{s_{\mathrm{NN}}}=200 \mathrm{GeV}$ and $U+U$ collisions at $\sqrt{s_{\mathrm{NN}}}=193 \mathrm{GeV}$, Physical Review Letters 123 (2019) $132302[1904.11658]$.

[22] J. Adam et al., Measurement of inclusive J/ $\psi$ suppression in Au+Au collisions at $\sqrt{s_{N N}}=200 \mathrm{GeV}$ through the dimuon channel at STAR, Physics Letters B 797 (2019) 134917 [1905.13669].

[23] X. Zhao and R. Rapp, Charmonium in medium: From correlators to experiment, Physical Review C 82 (2010) 064905 [1008.5328].

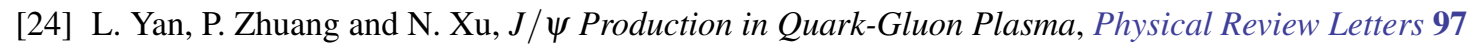
(2006) 232301 [0 608010$].$

[25] K. Zhou, N. Xu, Z. Xu and P. Zhuang, Medium effects on charmonium production at ultrarelativistic energies available at the CERN Large Hadron Collider, Physical Review C 89 (2014) 054911 [1401.5845].

[26] A. Andronic, P. Braun-Munzinger, K. Redlich and J. Stachel, Decoding the phase structure of QCD via particle production at high energy, Nature 561 (2018) 321 [1710.09425].

[27] B. Krouppa, A. Rothkopf and M. Strickland, Bottomonium suppression using a lattice QCD vetted potential, Physical Review D 97 (2018) 016017 [1710.02319].

[28] X. Du, M. He and R. Rapp, Color screening and regeneration of bottomonia in high-energy heavy-ion collisions, Physical Review C 96 (2017) 054901 [1706.08670]. 